\title{
Experimental Investigation of Transmission Coefficient of Reef Breakwaters
}

\author{
R.M.D.B. Rathnayaka, R.M.J.R. Rathnayaka and K.P.P. Pathirana
}

\begin{abstract}
A reef breakwater is a low crested, rubble mound breakwater without a conventional multilayered cross section. The performance of reef breakwaters has not been well researched in the past and the available research studies also do not produce conclusive results that could be used as design guidelines of these structures. It has been identified that transmission coefficient is one of the main parameters to quantify the performance of reef breakwaters. Accordingly, comprehensive laboratory investigations were carried out to study the transmission coefficient of reef breakwaters by varying different influence parameters. It was observed that wave steepness, crest width and depth of crest submergence are the most influential parameters on the transmission coefficient. The experimental data gathered from this study was used to check the performance of the existing equations developed to estimate the transmission coefficient of reef breakwaters. When using existing equations for the same input parameters, the estimated transmission coefficients appear to vary significantly from each other and hence, their applicability to real life problems is questionable. Therefore, an improved formula was developed to estimate the transmission coefficient of reef breakwaters using dimensional analysis that incorporates more influential parameters than those used in the existing equations. This formulation proved to be better than the previous equations.
\end{abstract}

Keywords: $\quad$ Reef breakwaters, Transmission coefficient, Physical model tests

\section{Introduction}

Reef breakwaters are low crested, rubble mound structures constructed using homogeneous piles of stones replacing the traditional multilayered cross sections. Because of the low crest height and the use of single armour layer, the reef breakwaters are relatively cheaper compared to the conventional rubble mound structures. Reef breakwaters are mainly used at places where partial attenuation of waves on the lee side of the structures is needed. The use of these breakwaters is now becoming popular among coastal engineers as one of the most effective types of shore protection structures. However, it is noted that there is a very little quantitative information available on reef breakwaters, particularly the design details.

Transmission coefficient which is defined in Eq. (1) is the important parameter governing the performance of reef breakwaters.

$K t=\frac{\text { Transmission wave height }}{\text { Incident wave height }}$

Very few studies on the transmission coefficient of reef breakwaters have been reported in literature. The estimation of transmission coefficient of reef breakwaters as suggested in CEM [1] is based on the results of different model tests and the applicability of this equation is rather limited. The method given by Ahrens, et al. [3] can be used for breakwaters with relative freeboard $\left(\frac{F}{H_{m o}}\right)$ less than 1 . The cross sectional area of the breakwater has been incorporated in this equation. Van der Meer, et al. [7] proposed an equation for the estimation of transmission coefficient for reef breakwaters having minimum and maximum transmission coefficient values of 0.075 and 0.8 , respectively. This equation also includes the surf similarity parameter. However, an important parameter like crest width of breakwater has not been considered in most of these methods while the range of applicability of these methods also is very limited. Van der Meer, et al. [8] has proposed an empirical equation to estimate transmission coefficient based on experimental studies using irregular wave conditions. In this equation, an attempt has been made to include the influence of crest width (B) and the permeability on the transmission coefficient introducing a constant in the formulation and

Eng. R.M.D.B. Rathanayaka, B.Sc Eng. (Hons.)

(Peradeniya), Eng. R.M.J.R Rathanayaka, B.Sc Eng. (Hons.) (Peradeniya), Faculty of Engineering, University of Peradeniya, Sri Lanka.

Eng. (Prof.) K.P.P. Pathirana, B.Sc. Eng. (Hons.) (Peradeniya), M.Eng., Ph.D. (KULeuven), C.Eng., FIE(Sri Lanka), MICE(London). Int.PE(SL), Professor of Civil Engineering, Department of Civil Engineering, University of Peradeniya, Sri Lanka. 
however, this has not shown any significant improvement in predicting accurate transmission coefficients for certain test conditions. In this research, the performance parameters of reef breakwaters were investigated under regular wave conditions and a new formulation was proposed for the transmission coefficient of reef breakwaters incorporating most of the parameters governing the transmission coefficient which will eventually contribute to improve the existing design guidelines.

\section{Methodology}

\subsection{Experimental Set-up}

The experiments were carried out in the wave channel in the Hydraulics Laboratory, Faculty of Engineering, University of Peradeniya. The wave channel is $12.75 \mathrm{~m}$ long, $0.52 \mathrm{~m}$ wide and $0.71 \mathrm{~m}$ deep. The side panels of the channel consist of sixteen $12 \mathrm{~mm}$ thick Perspex sheets, which enables visual observations such as wave breaking and interactions of waves with models constructed in the channel. The breakwater models were constructed using approximately uniformly distributed aggregates and two uniform sizes of aggregates.
One Armfield H40, resistant type, twin-wire wave probe was used to measure the wave parameters. Analog to Digital converter with 16 analog input channels was used to convert the analog signals from the wave probes to digital signals. LAB VIEW data acquisition software was used to acquire the data from wave probes.

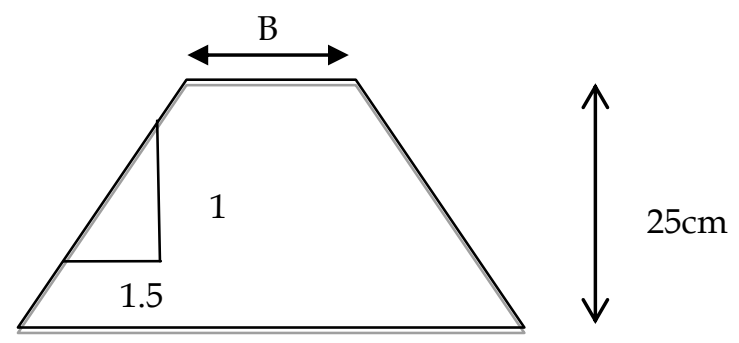

Figure 2 - Cross section of a model breakwater

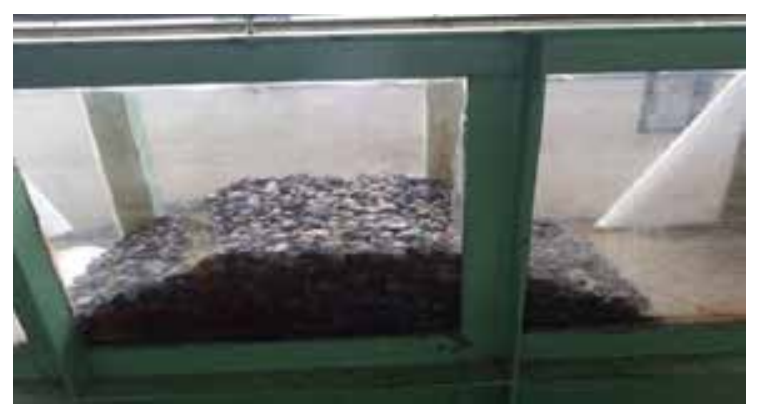

Figure 3 - Breakwater model of $40 \mathrm{~cm}$ crest width using $26 \mathrm{~mm}$ armour units

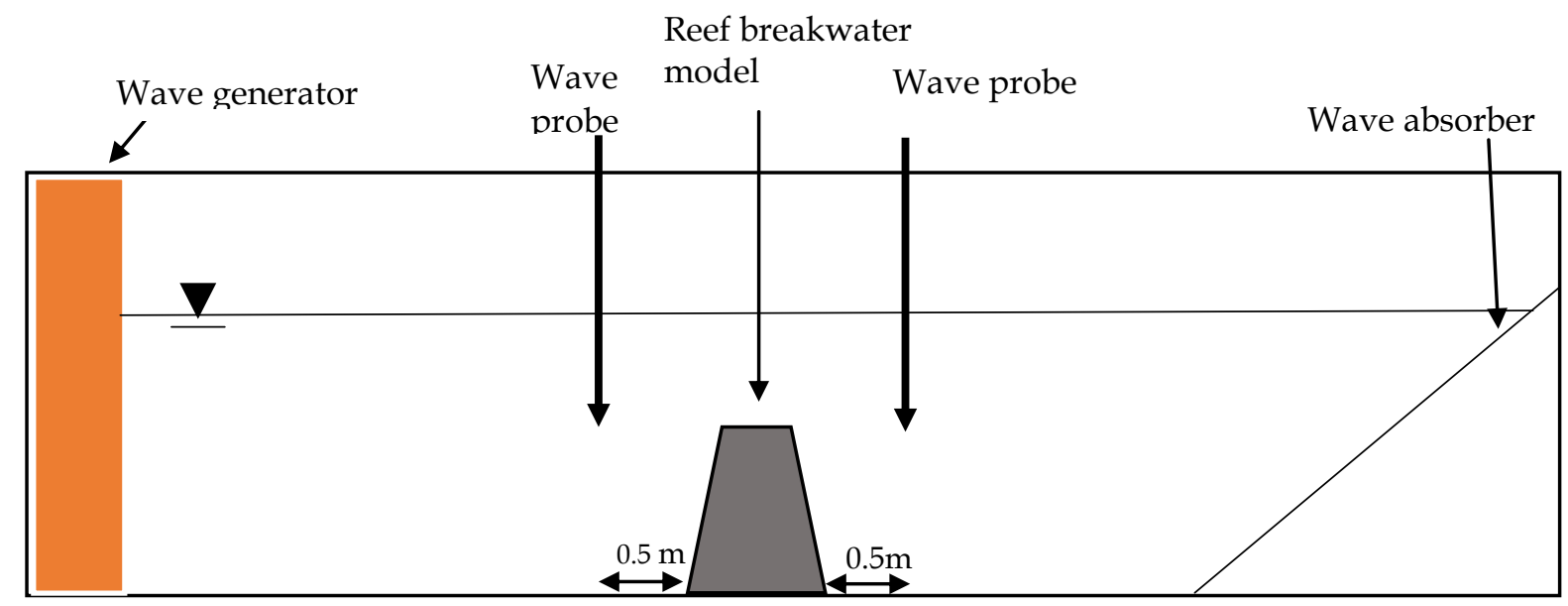

Figure 1 - Schematic diagram of the experimental set-up

(41.5 $\mathrm{mm}$ and $26 \mathrm{~mm}$ ) were used for the construction. For each stone class, three breakwaters were constructed with different crest width values (B) of $20 \mathrm{~cm}, 40 \mathrm{~cm}$ and 50 $\mathrm{cm}$. Trapezoidal cross sections were used to construct the breakwater models. Each breakwater model was constructed to a constant height of $25 \mathrm{~cm}$ and the side slopes of breakwater models were maintained at a constant slope of 1:1.5.
A MATLAB program was used to obtain the required wave parameters. Figure 1 shows the experimental set-up used in this study. 


\subsection{Model runs}

Table 1 shows the details of the experimental runs that were carried out by varying the model parameters. Though 192 experimental runs were conducted, few test runs could not be used for the data analysis as the measured wave heights on the leeside of the breakwater models in those test cases were small due to small amount of wave energy transmitted to the lee side of the breakwater. Rest of the experimental results were used in the data analysis.

Table 1- Different sets of experimental runs

\begin{tabular}{|c|c|}
\hline Variable & Values \\
\hline Wave height $(\mathrm{cm})$ & $2-17$ \\
Crest width $(\mathrm{cm})$ & $20,40,50$ \\
Water depth $(\mathrm{cm})$ & $25,30,35,40$ \\
Armour size $(\mathrm{mm})$ & $26,41.5$ \\
\hline \multicolumn{2}{|c|}{ Total No. of test runs $=192$} \\
\hline
\end{tabular}

\section{Data Analysis}

\subsection{Estimation of wave transmission coefficient using existing methods}

Each of the existing formulae were used to calculate the transmission coefficient using the experimental data obtained from this study and the calculated transmission coefficients were compared with the measured transmission coefficients. Figures 4 to 7 show the comparison of wave transmission coefficients estimated using the existing formulations with the measured values.

\section{a) Coastal Engineering Manual (CEM) [1]}

The equation given in the CEM for the calculation of transmission coefficient of reef breakwaters is given in Eq. (2).

$$
C_{t}=\left(0.031 * \frac{H_{s}}{D_{50}}-0.24\right) * \frac{R_{C}}{D_{50}}+b \quad \text { Eq. (2) }
$$

where, $C_{t}=$ transmission coefficient

for submerged breakwaters,

$b=-2.6 S_{o p}-0.05 \frac{H_{S}}{D_{50}}+0.85$

Eq. (3)

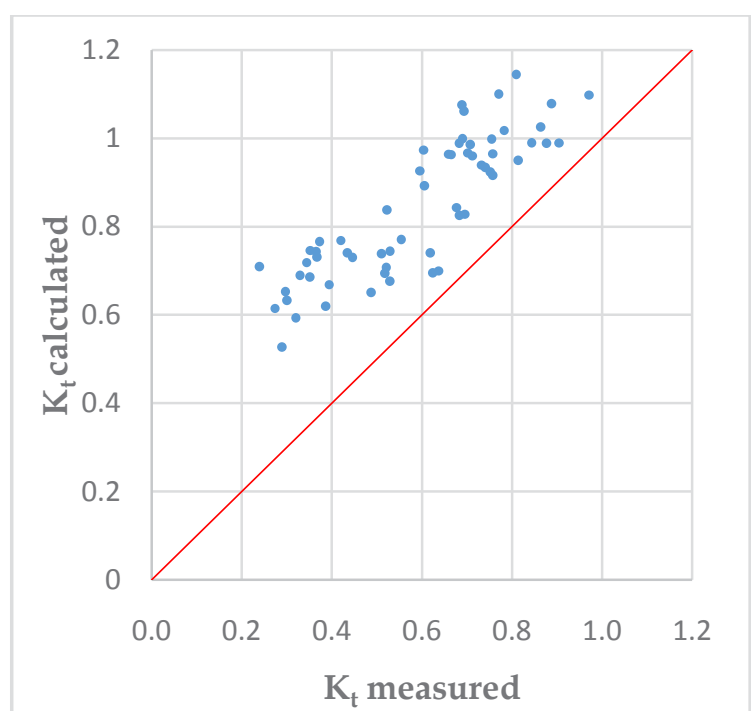

Figure 4 - Comparison of transmission coefficients estimated using Coastal Engineering Manual [1] with the measured values

As in Figure 4, it can be observed that data points are highly scattered. As almost all $K_{t}$ values estimated using Eq. (2) as per CEM are larger than the measured $K_{t}$ values, implying that CEM formula gives over predicted values for transmission coefficient. Some of the calculated transmission coefficients are greater than 1 which means the transmitted wave heights to the leeside of the breakwater are greater than incident wave heights. This is not acceptable.

b) Ahrens, et al. [3]

The equation proposed by Ahrens et al. [3] for the estimation of transmission coefficient of reef breakwaters with relative freeboard $\left(\frac{F}{H_{m o}}\right)$ less than 1 is given in Eq.(4).

$$
K_{t}=\frac{1}{1+\left(\frac{h_{c}}{d_{s}}\right)^{C_{1}}+\left(\frac{A_{t}}{d_{s} L_{p}}\right)^{C_{2}} \cdot \exp \left[C_{3}\left(\frac{F}{H_{m o}}\right)+C_{4}\left(\frac{A_{t}}{d_{50}^{2}} * \frac{1}{L_{p}}\right)\right]}
$$




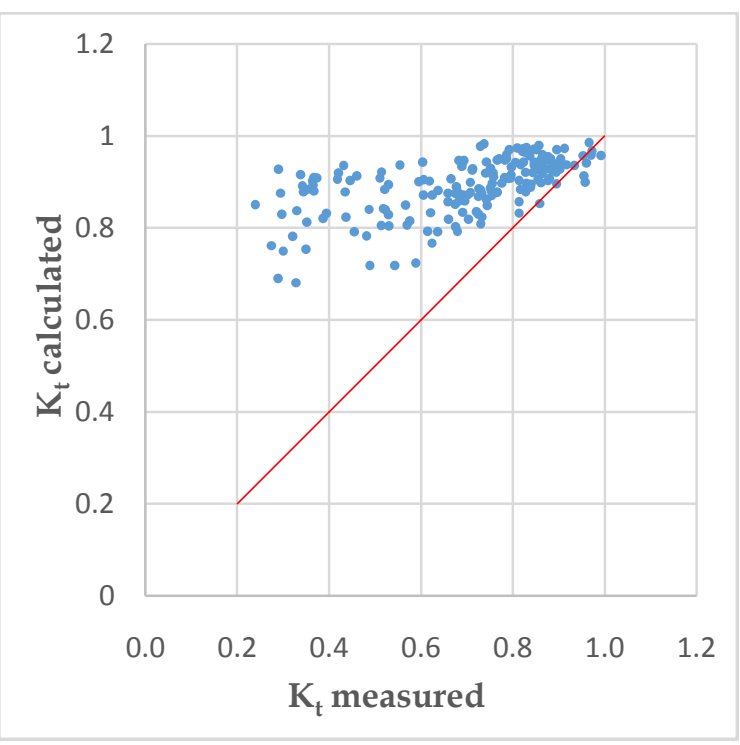

Figure 5 - Comparison of transmission coefficients estimated using Ahrens, et al. [3] with the measured values for $\frac{F}{H_{m o}}<1$

The comparison between the results from the formula and model test results are shown in Figure 5. As seen in the Figure, the transmission coefficients calculated from the equation given by Ahrens et al.[3] vary between 0.6 and 1, while the transmission coefficients obtained from the study vary within a wide range, viz. Between 0.3 and 1. When the freeboard is zero, there is a large deviation in the calculated and measured $K_{t}$ values.

Table 2 - Standard Deviation (SD) and Mean Relative Error (MRE) values for the calculated $K_{t}$ values.

\begin{tabular}{|c|c|c|}
\hline Parameters & MRE (\%) & SD \\
\hline$\frac{F}{H_{m o}}<1$ & 42.2 & 0.26 \\
\hline$\frac{F}{H_{m o}} \neq 0$ & 19.2 & 0.15 \\
\hline
\end{tabular}

According to the values in Table 2, the equation given by Ahrens et al. [3] for the estimation of $K_{t}$, gives better results for $\frac{F}{H_{m o}} \neq 0$ than for $\frac{F}{H_{m o}}<1$. The error of having $K_{t}$ values greater than 1 as noted in the previous comparison is not seen in this equation. c) Van der Meer et al. [7]

Van der Meer et al. [7] proposed the following equation (Eq. 5) for the calculation of transmission coefficient of reef breakwaters with minimum and maximum transmission coefficient values of 0.075 and 0.8 , respectively.

$$
K_{t}=\left(-0.3 \frac{R_{c}}{H_{o m}}+0.75\left[1-\exp \left(1-0.5 \varepsilon_{o p}\right)\right]\right)
$$

For $-1.66<\frac{R_{C}}{H_{o m}}<1.66$

The formula does not include the crest width of break water and the armour gradation. Several data sets from the present study could not be used here, as some of the data sets exceed the applicability range defined for Eq. (5). For some data sets, $K_{t}$ values are higher than unity suggesting that transmitted wave height is greater than the incident wave height which is not acceptable.

In this study, only few parameters were considered in deriving the equation for the transmission coefficient. Surf similarity parameter and seaward slope of the breakwater are incorporated in the equation. In the present study, seaward slope of the reef breakwater was kept constant throughout the experimental procedure.

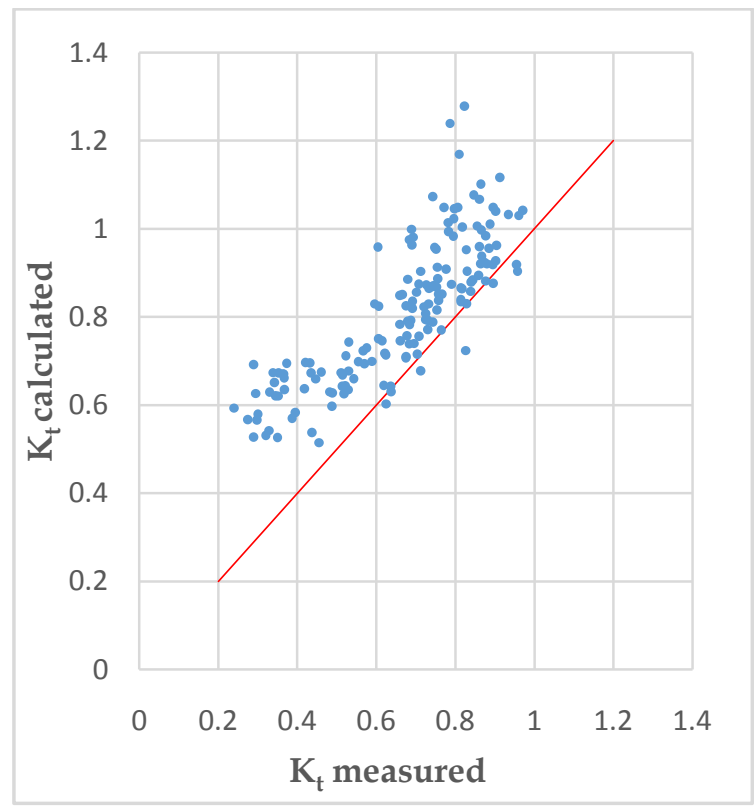

Figure 6 - Comparison of transmission coefficients estimated using Van der Meer et al. [7] with the measured values 
d) Van der Meer et al [8]

Combination of the research findings on the transmission coefficient of reef breakwaters proposed by Van der Meer, D' Agremond, and de Jong [8], an empirical equation (Eq.6) was proposed to calculate transmission coefficient based on the experimental results obtained using irregular wave conditions.

$K_{t}=-0.4 \frac{R_{c}}{H_{m o}}+\left(\frac{B}{H_{m o}}\right)^{-0.31}\left[1-\exp \left(-0.5 \epsilon_{o p}\right]{ }^{*} C\right.$

Eq.(6)

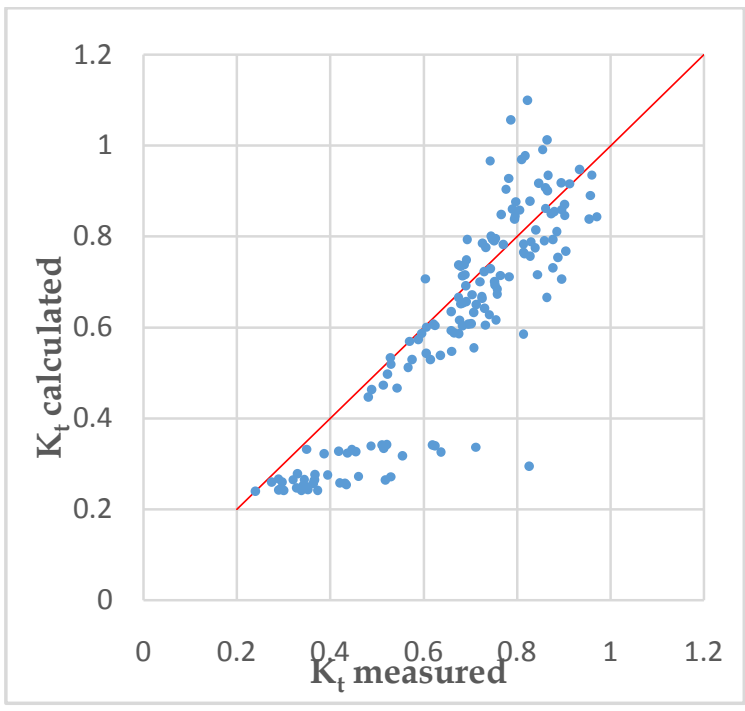

Figure 7 - Comparison of transmission coefficients estimated using Van der Meer et al [8] with the measured values

The comparison between the calculated $K_{t}$ using Eq. (6) and the measured $K_{t}$ is shown in Figure 7.

Two separate data groups can be seen in Figure 7. One data set is somewhat scattered around $45^{0}$ line while the other group shows a significant deviation between the measured $K_{t}$ and the estimated $K_{t}$ values. The data set that has a significant deviation between the estimated and measured values are corresponding to the zero depth of crest submergence $\left(R_{c}=0\right)$.

For $R_{c}>0$, this equation estimates $K_{t}$ values with a mean relative error of $5.77 \%$ which is a significant improvement from the equations previously discussed. In conclusion, Eq. (6) gives a better estimation of $K_{t}$ than the previously presented equations when breakwater is totally submerged, but it gives poor results when the water level and crest of the breakwater are at same level.

From the analysis, it can be seen that the data points are highly scattered. In addition, there are few test cases where the estimated transmission coefficients are greater than one, which is not acceptable.

It seems that there are major drawbacks and limitations in the existing equations. In order to quantify the errors given by the formulae suggested in previous studies, the standard error parameters such as Mean Relative Error (MRE) and Standard Deviation (SD) were calculated (Table 3).

Table 3 - Standard deviation and MRE values for the $K_{t}$ values calculated from previous studies

\begin{tabular}{|c|c|c|}
\hline Study & MRE (\%) & SD \\
\hline $\begin{array}{c}\text { Coastal } \\
\text { Engineering } \\
\text { Manual [1] }\end{array}$ & 53.0 & 0.27 \\
\hline $\begin{array}{c}\text { Ahrens, et al. [3] } \\
\text { Van der Meer, et } \\
\text { al. [7] }\end{array}$ & 25.2 & 0.26 \\
\hline $\begin{array}{c}\text { Van der Meer, et } \\
\text { al. [8] }\end{array}$ & 12.6 & 0.17 \\
\hline
\end{tabular}

Based on the observations from Figures 4 to 7 and MRE and SD values, it can be concluded that the existing formulations derived from previous studies for the calculation of the transmission coefficient are not accurate enough. Among the studies considered here, the equation given by Van der Meer, et al. [8] is the most accurate one with a MRE of $12.6 \%$. Hence plenty of space and necessity can be identified for a more improved formula to calculate the transmission coefficient. 


\subsection{Development of a new formula to calculate the Transmission coefficient}

The selection of governing parameters affecting the transmission coefficient was done with the help of coastal engineering concepts and past studies.

Following parameters were selected as the governing parameters associated with the performance of reef breakwaters considering the transmission of waves.

$$
K_{t}=f\left(H_{s}, d_{s}, d, B, D_{50}, T, \tan \alpha, g\right)
$$

where, $H_{s}$ is the significant wave height, $d_{s}$ is the depth of crest submergence, $d$ is the water depth, $B$ is the crest width, $D_{50}$ is the medium armour size, $T$ is the wave period and $\tan \alpha$ is the seaward slope. 'Buckingham Pi Theorem' was used to formulate dimensionless parameters. $H_{S}$ and $T$ were used as repeating variables which had independent dimensions and the following dimensionless groups were formed.

$$
K_{t}=f\left(\frac{H_{s}}{d}, \frac{H_{s}}{d_{s}}, \frac{H_{s}}{B}, \frac{H_{s}}{D_{50}}, \frac{g T^{2}}{H_{s}}, \tan \alpha\right)
$$

Since the seaward angle of the model breakwaters were maintained at a constant slope of $1: 1.5$, the term $\tan \alpha$ was not considered when forming the formula. Therefore, the following dimensionless parameters were used to formulate the relationship.

$$
K_{t}=f\left(\frac{H_{s}}{d}, \frac{H_{s}}{d_{s}}, \frac{H_{s}}{B}, \frac{H_{s}}{D_{50}}, \frac{g T^{2}}{H_{s}}\right)
$$

Multi variable regression analysis was carried out to obtain the relationship among these dimensionless groups. (Coefficients were found using regression function embedded in MS Excel using the experimental data obtained in this study). The derived formula in this study is shown in Eq. (7).

$$
\begin{aligned}
& K_{t}=0.613-1.83\left(\frac{H_{i}}{d}\right)^{2}+0.02\left(\frac{H_{i}}{B}\right)^{-1}+0.317\left(\frac{d_{s}}{H_{s}}\right)^{0.35} \\
&+ 0.002\left(\frac{H_{i}}{D_{50}}\right)^{2}+5.32 * 10^{-7} *\left(\frac{g T^{2}}{H_{i}}\right)^{2}
\end{aligned}
$$

Eq. (7)

After deriving the formula, $K_{t}$ values calculated from the formula was plotted against the $K_{t}$ values obtained from the experimental runs as shown in Figure 8. Eq. (7) appears to predict reasonable values of $K_{t}$ compared with the previous methods. The transmission coefficients were estimated corresponding to experimental data from present study with a MRE value of $9.13 \%$ from this equation.

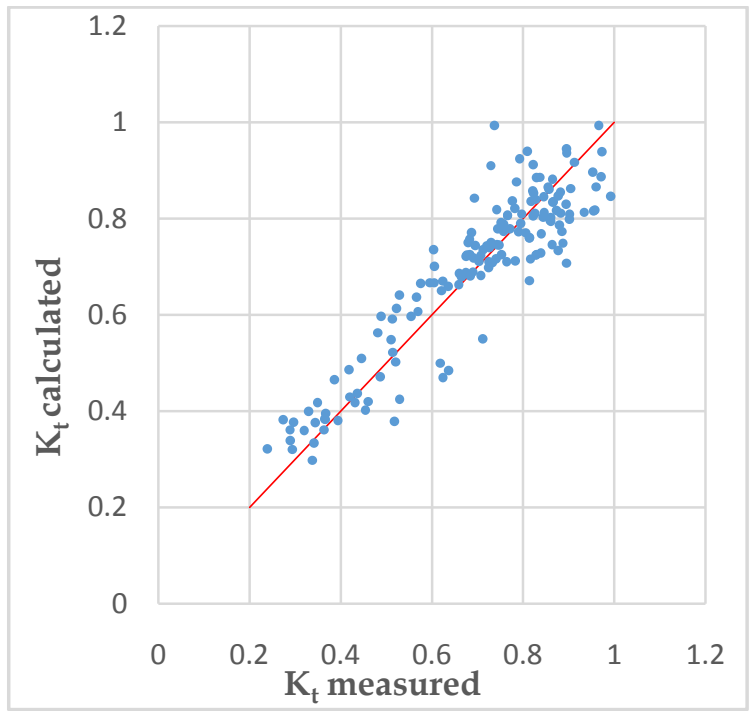

Figure 8 - Comparison of experimental results with calculated values with the proposed formula.

\section{Conclusions and Recommendations}

The formula derived for the estimation of transmission coefficient in the present study contains more variables than the existing formulae. Existing equations are poor in estimating transmission coefficient when the depth of crest submergence is zero. However, this shortcoming was eliminated in the equation proposed in this study. The limitations of this study are given below; Present study was done using regular wave conditions.

- The effect of seaward slope of breakwater is not investigated during this study as it was maintained at a constant value of 1:1.5.

- Wave periods used for the tests vary from 0.7 to 1.25 seconds. For higher wave periods, the present equation should be validated.

- Only two armour sizes were used for the construction of the breakwaters. 
Considering the limitations of the present study and other factors, a number of recommendations to improve the results of the study can be suggested.

- Increasing the range of wave heights and wave periods in order to increase the limit of applicability.

- Construction of model reef breakwaters with varying seaward slopes.

- Use of irregular and oblique waves. and

- Conduct tests with various sizes of armor units.

\section{References}

[1]. Coastal Engineering Manual, 1110-2-1100 U.S. Army Corps of Engineers, 2001.

[2]. Ahrens, J. P., 1984. 'Reef Type Breakwaters', Coastal Engineering Journal, ASCE, New York.

[3]. Ahrens, J. P., 1987. 'Characteristics of reef breakwaters', Coastal Engineering Research Center.

[4]. Ahrens, J. P., 1989. 'Stability of Reef Breakwaters', Journal of Waterway, Port, Coastal, and Ocean Engineering, Vol. 115, no. 2, pp. 221234.

[5]. Ahrens, J. P., Cox, J., 1990. ‘Design and Performance of Reef Breakwaters', Journal of Coastal Research, no.7, pp. 61-75.

[6]. Dattari, J., Raman, H., Shankar, N.J., 1978. 'Performance characteristics of submerged breakwaters', Coastal Engineering Journal, ASCE, New York, pp. 2152-2171

[7]. Van derMeer, J. Wang, B., Wolters, A., Zanuttigh, B., Kramer, M., 2003. 'Oblique Wave Transmission over Low-Crested Structures'. ASCE, Proc. Coastal Structures, Portland, Oregon.

[8]. Van der Meer, J. W. and d'Angremond, K., 1991. Wave transmission at low-crested structures, ICE, Thomas Telford. In: Coastal structures and breakwaters, London, United Kingdom, p. 25 - 40. 